\title{
Rechtsgeschichte
}

http://www.rg-rechtsgeschichte.de/rg6

Zitiervorschlag: Rechtsgeschichte Rg 6 (2005)

$\operatorname{Rg} 62005$

$211-216$

http://dx.doi.org/10.12946/rg06/211-216

\section{Jakob Tanner}

Body-Shaping 
von den Herausgebern als eigenständig behandelten England wandelt sich somit unter dem Einfluss europäischen Rechtes erheblich.

Diese kritischen Anmerkungen ändern indessen nichts daran, dass es sich bei dem Dictionnaire de la culture juridique insgesamt um einen großen Wurf handelt, der Juristen wie juristisch interessierte Laien in gleicher Weise in seinen Bann ziehen kann. Es ist zu wünschen,

\section{Body-Shaping *}

Menschen haben einen Körper - und zugleich sind sie ihr Leib. Dieses Spannungsfeld zwischen Haben und Sein lässt sich auflösen, wenn der Körper als konstitutiver Bestandteil einer Person und damit als materielle Essenz personaler Identität aufgefasst wird. Die Theorie der self-ownership sieht in diesem Grundgefühl den Ausdruck eines possessiven Individualismus, aus dem sich wiederum ein expliziter Rechtsanspruch ableiten lässt: Als Besitzer meiner Person und ihrer Fähigkeiten gehört mir auch mein Körper und dessen Leistungen. Ich kann - vorausgesetzt, andere kommen dadurch nicht zu Schaden - das ganze Potential, das in meiner Person steckt, ungehindert nutzen und beliebig verwerten. Es ist nicht erstaunlich, dass dieses Konzept, das man in heutiger Terminologie eine "Ich-AG « nennen könnte, im Zuge der Entfaltung der bürgerlichen Gesellschaft aufkam; in Großbritannien wurde die Freiheit des Bürgers I 679 mit der Habeas-Corpus-Akte sanktioniert, der auf den lebenden, d.h. den lebendgeborenen Körper Bezug nahm und den Grundrechten einen Körperstatus zumaß. In John Lockes »Abhandlungen über die Regierung « findet sich der dass ein vergleichbares Wörterbuch des Rechtsdenkens eines Tages auch für den deutschsprachigen Leser geschaffen wird. Die Erarbeitung eines solchen Werkes böte eine gute Gelegenheit für eine Rückbesinnung auf die Grundlagen unseres Rechts in einer Zeit zunehmender Geschichts- und Theorievergessenheit der Juristen.

Achim Seifert prägnante Satz, jeder Mensch verfüge über das »Eigentum an seiner eigenen Person ", woraus wiederum folge, dass »die Arbeit seines Körpers und das Werk seiner Hände [...] im eigentlichen Sinne sein Eigentum « seien. Damit wird - auch wenn self-ownership nicht mit body-ownership gleichgesetzt werden kann - dennoch eine stabile Beziehung zwischen Körper und Recht gestiftet, die bis heute ihre Suggestivkraft nicht eingebüßt hat und auch zur Abwehr egalitärer, auf Verteilungsgerechtigkeit abzielender Postulate eingesetzt wird.

Der von Ludger Schwarte und Christoph Wulf herausgegebene Sammelband »Körper und Recht " entfaltet - so der Untertitel - »anthropologische Dimensionen der Rechtsphilosophie « in einer Weise, dass solche kurzschlüssige Thesen in Frage gestellt werden können. Die Auswirkungen der Biowissenschaften und neuer medizinischer Techniken auf Rechtsentwicklung und -anwendung kommen in diesem Band ebenso ausführlich zur Sprache wie das Problem eines Rechtssystems bzw. Moralverständnisses, das auf Menschen beschränkt ist und sich mit dem Vorwurf des Speziezismus und einer anthropo-

\footnotetext{
* Ludger Schwarte, Christoph Wulf (Hg.), Körper und Recht. Anthropologische Dimensionen der Rechtsphilosophie, München: Wilhelm Fink 2003, 427 S., ISBN 3-7705-3905-2
} 
zentrischen Arroganz auseinanderzusetzen hat. Dabei sind Überlegungen und Theoreme einer nichtnormativen, sich für den wandelbaren, historisch und kulturell geprägten menschlichen Körper interessierenden Anthropologie maßgebend, wie sie vom Interdisziplinären Zentrum für Historische Anthropologie der Freien Universität Berlin schon seit einiger Zeit diskutiert werden. Einleitend wird die Frage, ob und welche Rechte der Körper hat oder haben könne, aufgegriffen und zugleich auch die metaphorischen Operationen, mit welchen der Körperbegriff auf rechtliche »Körperschaften « übertragen werden kann, angesprochen. Die einprägsamste Figur ist hier der Hobbessche Leviathan, welcher den Staat als die absolute Verkörperung eines Rechts darstellt, das wiederum durch die Bürger inkorporiert werden muss, um die Ausübung verrechtlichter Macht zu gewährleisten. Im Zuge der Aufklärung wurde der Rechtspositivismus, der alles geltende Recht an den Willen eines Souveräns und damit an Herrschaftsstrukturen zurückbindet, durch das Naturrecht kritisiert, welches die Würde des Menschen aus seinem Vernunftcharakter herleitet. Die beiden Herausgeber brechen diese dichotome Entgegensetzung einer naturrechtlichen und einer rechtspositivistischen Position durch den Rekurs auf Hans Kelsens Vorstellung auf, Recht sei weder eine anthropologische Universalie noch ein schierer Machteffekt. Damit gerät der reflexive Begründungszusammenhang einer $»$ Rechtlichkeit von Rechten " ins Zentrum der Argumentation. Mit der » Verschiebung von der universellen Vernunft zu dem besonderen Wert menschlichen Lebens" dient nun die Lebendigkeit des konkreten menschlichen Körpers zur Begründung von Recht, womit sich eine "Verzahnung von Anthropologie und Rechtswissenschaft « ergibt. Die Herausgeber bezeichnen allerdings diese Argu- mentationsfigur einer Konstruktion des Konzepts "Mensch « aus Rechtsbegriffen und einer Modellierung des Körpers durch juridische Diskurse als "fatal «, sehen aber auch in der Antithese einer Emergenz von Recht aus dem Körper bzw. aus einer verkörperten Person keine valable Alternative. Weiterführend scheint es dann, vom Körper als dem »Anderen des Rechts « zu sprechen. Körperliche Präsenz entzieht sich aus dieser Sicht der Vereinnahmung durch Recht, während umgekehrt rechtliche Normen gegenüber dem Körper kontingent blieben. So gilt es denn, einen Blick »hinter das kodifizierte Rechtssubjekt « auf jene kulturellen Praktiken und Inszenierungsformen $\mathrm{zu}$ werfen, die zusammenfassend mit dem forschungsprogrammatischen Begriff »Performativität des Rechts « bezeichnet werden.

Die 2I Beiträge des interdisziplinär konzipierten Bandes, deren Autoren vor allem in der Philosophie, der Rechtswissenschaft und den Kulturwissenschaften angesiedelt sind, teilen sich in drei Schwerpunkte auf: Körperinszenierungen und Repräsentation des Rechts (I), Biotechnologie und Rechte des Körpers (II) sowie Körper und Souveränität (III). Teil I enthält Aufsätze, die unter dem Leitbegriff »Performanz des Rechts « Körperinszenierungen und Repräsentationen des Rechts in unterschiedlichen historischen und kulturellen Kontexten analysieren. Körper und Recht werden dabei über den Begriff der Inkorporation aufeinander bezogen. Normen, Werte und Rituale formen alltägliches Handeln, wobei das praktische Wissen, das diesen performativen Äußerungen zugrunde liegt, in den Akteuren selbst verkörpert ist und in seinem permanenten Gebrauch immer wieder konkretisiert und auch modifiziert wird. Christoph Wulf zeigt in einem instruktiven Beitrag über »Ritual und Recht«, wie die Einschreibung 
von Normen in individuelle Körper komplexe Rituale öffentlichen Charakters entstehen lässt, die auf szenischen Arrangements menschlicher Körper beruhen. Über solche Inszenierungen wird die gesellschaftliche Ordnung nicht nur stabilisiert, sondern sie gerät auch in eine Veränderungsdrift. Rituale sind somit nicht nur auf selbstidentische Reproduktion von Verhaltensweisen, sondern intrinsisch auf Wandel hin angelegt. Gerichtsprozesse können als ritualisierte Interaktionsformen analysiert werden, die auf einer öffentlichen Bühne inszeniert werden. Als performative »Gesamtkunstwerke « beruhen sie ebenso auf choreographischen Mustern wie in der Materialität des Raumes, in dem sich diese entfalten. Die Verkettung von Handlungsabläufen in vermachteten Raumstrukturen setzen dabei den Körper als ein Gedächtnis in Bewegung, welches praktisches Wissen mimetisch exprimiert. Mimesis - ein Zentralbegriff der Historischen Anthropologie Wulfscher Prägung - bezeichnet allerdings nicht nur diese Nachahmung, sondern auch die ihr inhärenten kreativen, aus dem bloßen Nachvollzug gesetzter Normen herausspringenden Momente.

Ein solches Konzept von Mimesis verwendet auch Gunter Gebauer in seiner Analyse der Inszenierung von Regelverstößen und Regeln im Sport. In einer originellen Nietzsche-Interpretation weist der Autor auf die Inkorporationsgewalt hin, die aus Menschen ein ambulantes Gedächtnis der Gesellschaft macht. Damit gelingt es, von einer Vorstellung von Regeln wegzukommen, welche diese zu virtuellen Kraftlinien hypostasiert, die auf wundersame Weise in einem sozialen Raum gleichsam zwischen den Menschen existieren. Anstatt die verhaltensprägende Potenz der Regel als etwas zu denken, was von außerhalb auf Akteure einwirkt, wird diese in den menschlichen Körper hinein verlagert und als materialisierte Disposition aufgefasst. Sportliche Inszenierungen bieten weiten Raum für die Untersuchung, wie dieses in den menschlichen Körper implementierte Regelgedächtnis situationsspezifisch aktiviert wird. Dabei lässt sich eine Transformation des Spielsubjekts in Richtung eines listigen Umgangs mit Regeln erkennen, was z. B. dazu geführt hat, dass moderne Fussballspiele seit den I97oer Jahren zunehmend zu einem »Kampf um Interpretationen « wurden, in denen sich Metis, d.h. die Fähigkeit, regelkonforme Verhaltenserwartungen überraschend zu überlisten, als spielentscheidender Faktor erweisen kann. »Das war die Hand Gottes «: in diesem expost-Kommentar Diego Maradonas zu einem regelwidrig mit der Hand erzielten, eventuell spielentscheidenden Tor zeigt sich diese aus dem Zusammenspiel von Mimesis und Metis resultierende Ausweitung der Grauzone.

Die in Teil II des Buches versammelten Aufsätze verschieben die Problematik des Zusammenhangs von Körper und Recht auf ganz andere Problembereiche und thematisieren mehrheitlich die "anthropotechnische Wucht der neuen Biowissenschaften «. Stephan Rixen, von dem diese Formulierung stammt, analysiert in seinem Beitrag zur »reprogenetischen Diffusion des Körpers « den impact, den diese Auflösungstendenz und der damit einhergehende Konturverlust von Körperbildern auf das Recht haben. Der Autor zeigt plausibel, dass die Verbindung von Menschenrechten und menschlichem Körper ein interpretativer (und kein deskriptiver) Akt ist und wendet sich dann der grundrechtlichen Relevanz der zunehmenden Grenz- und Zweifelsfälle am Lebensanfang und -ende zu. Es wird deutlich, dass die Definition der »Menschenwürde« im Grundgesetz mit guten Gründen offen gehalten wurde; anstatt sich auf eine Wesenbestimmung festlegen $\mathrm{zu}$ wollen, wurde 
ein »offenes Menschenbild « postuliert und bloß eine ordinale Prioritätenordnung festgelegt, die dem Individuum einen absoluten, d.h. einer Güterabwägung nicht zugänglichen Vorrang vor staatlichen Interessen zumisst. Damit erweist sich das Grundgesetz zwar als körperbezogen, aber eben nicht - wie von Kritikern unterstellt als substanziell biologistisch. Rixen leitet daraus das Plädoyer ab, »das positive Verfassungsrecht so fortzubilden, dass seine basale Referenz auf Menschen-Körper unter dem Druck biowissenschaftlicher Veränderung erkennbar bleibt «.

Einer ähnlichen Ausgangslage ist Beate Herrmann verpflichtet, welche das für ihren Aufsatz titelgebende Konzept einer self-ownership kritisiert. Die Autorin geht von der Feststellung einer Ambivalenz aus: Einerseits zeichnet sich seit einiger Zeit eine zunehmende Verwertbarkeit des menschlichen Körpers im Zeichen der Reproduktions- und Transplantationsmedizin ab, bis hin zur These einer "Sozialpflichtigkeit « des menschlichen Körpers. Andererseits lässt sich ein fundamentaler Widerstand gegen die Tendenz erkennen, den Körper den Prinzipien der Verteilungsgerechtigkeit zu unterwerfen oder ihn als eine kommodifizierte Ressource, deren Nutzung den Regeln des Marktes gehorcht, zu behandeln. Diese Position, welche die Autorin in Auseinandersetzung mit Kant entwickelt, besticht durch ihre Kritik an der problematischen Gleichsetzung von Körper und Person und die Fähigkeit, das menschliche Körpersein (die Binnenperspektive der Wahrnehmung) durch die Einsicht zu ergänzen, dass Menschen durchaus einen Körper haben (die Außenperspektive der Betrachtung). Somit wird es der menschlichen Person, die zunächst vom Eigenwert ihres Körpers ausgeht, qua intellektueller Kapazitäten auch ermöglicht, sich zum eigenen Körper in ein instrumentelles Verhältnis zu setzen, ihn zu objektivieren und insoweit zu einer Sache zu machen. Mit dieser differenzierten Sicht sind zwar die Probleme, die sich aus biomedizinischer Sicht stellen, alles andere als gelöst, es lässt sich aber ein produktiver Ausgangspunkt für ihre Diskussion bestimmen, der a priori-Engführungen auf die eine oder andere Seite vermeidet. Ebenso schwebend in der Konklusion ist der Beitrag von Ulrich Steinvorth, der in Auseinandersetzung mit der Lockeschen These der self-ownership und dem Kantschen Verbot, den Menschen als ein Instrument zu benutzen (das zusammenfällt mit der These, ein Mensch sei ein Selbstzweck), die Legitimität des Klonens untersucht und zeigt, wie wenig die kategorischen Argumente, die Hans Jonas und Jürgen Habermas dagegen entwickeln, geeignet sind, aus den normativen Aporien der modernen Biomedizin herauszuführen - wobei offen gelassen wird, ob dies überhaupt erstrebenswert ist bzw. möglich sein soll.

Teil III des Buches greift zentrale Aspekte des zweiten Teils nochmals unter anderen Vorzeichen auf. In Auseinandersetzung mit Argumenten, wie sie von Kritikern der speziezistischen Verengung des Begriffs der »Menschenwürde«, aber auch von Protagonisten einer neuen Bioethik vorgetragen werden, insistiert Heike Baranzke auf der Validität der Kantschen Handlungsanweisung, die postuliert, man solle die Menschheit sowohl in seiner eigenen Person wie auch in der Person eines jeden anderen »jederzeit zugleich als Zweck, niemals bloß als Mittel « brauchen. Indem die Autorin zum Schluss gelangt, »nur eine Rechtsphilosophie, die sich von der Person und nicht vom Körper herleitet «, vermöge dem Kantschen Postulat Nachachtung zu verschaffen, stellt sie nochmals akut die Frage nach der Beziehung zwischen dieser Person und ihrem Körper; mit der These, dass für Kant - entgegen einem weit verbreiteten 
Vorurteil - der Mensch keinesweg ein »körperloses moralisches Subjekt, sondern verkörperte Persönlichkeit ist «, wird eine, allerdings seit langem in Diskussion sich befindliche Möglichkeit eröffnet, die Dignitas-Würde der Menschen und die Bonitas-Würde der Tiere in eine neue Synthese zu bringen. In einem Akt menschlicher Selbstverpflichtung sollte es - »in Ansehung « der Tiere - darum gehen, »der eigenen Menschenwürde in einem Akt positiver rechtlicher Anerkenntnis einer Tierschutzpflicht Ausdruck zu verleihen «. Der Vorteil dieser Position besteht darin, einer Biologisierung des Menschen zu entsagen. Sie vermag jedoch die in Anlehnung an Hans Kelsen und andere entworfene Argumentation jenseits einer naturrechtlich-vernunftbegründeten moralischen Subjektphilosophie nicht weiterzudenken und fällt damit in jene speziezistische Kategorie der »Gattungswürde « zurück, die einleitend als Problem erkannt wurde.

Direkter auf die Frage nach dem Zusammenhang von Souveränität, Körper und Recht geht der Beitrag von Jörn Ahrens ein, der - auf Michel Foucault und Giorgio Agamben Bezug nehmend - die "Konflikte um die Sterbehilfe « nachzeichnet. In traditionalen Herrschaftssystemen war der Tod ein Dispositiv der Macht; das Verfügenkönnen über Leben und Tod gehörte zur normativen Signatur der Souveränität. Mit der industriegesellschaftlich-bürgerlichen Moderne ergab sich eine folgenreiche Verschiebung weg von der Drohung des Souveräns mit dem Mord und hin zur Verantwortung der Macht für das Leben. Diese "Biomacht « - und die darauf basierende "Biopolitik « - gehen, so Foucault, aus dem Willen zur intensiven Ausschöpfung der Lebenskapazitäten durch Technologien zur Optimierung von individuellen Körpern hervor. Letztere werden auf die gesellschaftlichen Pro- duktivitätsnormen eines Metakörpers hin einreguliert, aus- und zugerichtet. Der Souveränitätsdiskurs des Todes kristallisierte sich unter diesen Bedingungen einer produktiven Wechselwirkung von Wissen und Macht zunehmend am »falschen « Körper, der aufgrund seiner Dysfunktionalität eine Gefahr für das Machtdispositiv ist und der deshalb - mit unterschiedlicher Systematik und Brutalität - ausgeschaltet wird. Sterbehilfe, verstanden als »ein genuin subjektives Autonomiemoment «, liegt nun quer zu diesem Souveränitätsdiskurs; indem die Sterbehilfe »den Tod an das Subjekt zurückgeben " möchte, macht sie die Unterscheidung von »sterben lassen « und »leben machen « unkenntlich. Der Autor zeigt die disparate Stellung des Todes zum Recht am Beispiel jener Menschen auf, die, weil irreversibel bewusstlos, zu keiner Kommunikation mehr fähig sind und deren passiver Körper zum Indikator für die Überschreitung der Grenze zwischen Leben und Tod geworden ist. In dieser Situation wird die Politik der Sterbehilfe durch die Zuschreibung eines Willens von Seiten sozial Bevollmächtigter bestimmt. Sie passt sich somit in das Kontinuum zur körperlichen Domestizierung und Disziplinierung des Körpers ein; indem sie aber auf einer Abstraktion von der Körperlichkeit beruht, substituiert sie diese durch den Willen, der damit als »das entscheidende Medium des Rechts « erscheint.

Die vorgestellten Beiträge zeigen, dass der Sammelband heterogen aufgebaut ist. Vor allem in Teil zwei und drei herrscht ein beträchtliches kompositorisches Durcheinander. In den Katarakten zwischen unterschiedlichen Argumentationsfiguren stürzen nicht wenige wichtige Gedanken ab. Insbesondere die Brauchbarkeit der Kantschen "Zweck-Mittel-Formel « wird aus sehr verschiedenen Perspektiven diskutiert, ohne dass deren Implikationen etwas systematischer 
herausgearbeitet würden. Die Konjunktion im Buchtitel spannt zwar einen interessanten Problemhorizont auf - man ist allerdings nach der Lektüre des Buches einmal mehr geneigt, von der Verklammerung zweier Zentralbegriffe mit einem "und " in Buchtiteln dringend abzuraten. Zudem weckt die Formulierung "Körper und Recht « auch Erwartungen, die nicht eingelöst werden. Als ob das Hirn nicht zum Körper gehören würde, fehlt eine Auseinandersetzung mit den wichtigen Diskussionen um die menschliche Willensfreiheit und die rechtlichen Konsequenzen, die aus neurowissenschaftlichen Forschungen allenfalls - oder eben gerade nicht - zu ziehen wären. Nicht nur in Teil I sind zudem die Medien merkwürdig abwesend - dies obwohl »Körperinszenierung und Repräsentation des Rechts « sich ebenso wenig von einer massenmedialen Dimension abtrennen lassen wie die Frage des Zusammenhangs zwischen Souveränität und Körper. Der Beitrag von Jacques Poulain über die magische Meisterung von wirtschaftlichen und sozialen Krisen durch die Verabsolutierung von »Nation und Rasse « im Europa des ausgehenden 20. Jahrhunderts ist in der Druckversion kaum nachvollziehbar. Umgekehrt gibt es den plastisch geschriebenen Text von Wilhelm Schmidt-Biggemann zu Joseph de Maistres Straf- theorie. Hier wird gezeigt, wie de Maistre in Auseinandersetzung mit der Französischen Revolution, die er in der Tradition der Herrschaft des Bösen ansiedelt, das Problem der Theodizee neu aufrollt. Was etwa über den »Fanatismus der Revolutionssoldaten " und die »Christologie des Opfers « gelesen werden kann, ist erhellend - die Vorstellung des Henkers, der als Repräsentant der Macht seines blutigen Amtes waltet, genügt jedoch nicht, um diesen Text in die Problemstellung des Sammelbandes zurückzubringen.

Die monierte Heterogenität kann auch positiv gewertet werden. In einer Phase, in der Grundlegendes neu überdacht werden muss, lässt sich kein Sammelband konzipieren, der mit dem Anspruch auftritt, die zentralen Aspekte und Problemstellungen in sich $\mathrm{zu}$ vereinigen. Wer diesem Wunsch nach einer umfassenderen thematischen Inkorporation nicht nachgeben will, dem oder der präsentiert sich eine Publikation, die interessante Denkanstöße gibt - nicht zuletzt auch durch die Konfrontation des »stummen Körpers ", wie er in der Einleitung programmatisch beschworen wird, mit den rechtlich beredten Körpern, die in vielen Beiträgen des Sammelbandes Gestalt annehmen.

Jakob Tanner

\section{Il manganello e la bilancia*}

Lo studio del ventennio fascista da parte della letteratura giuridica italiana ha tradizionalmente costituito l'occupazione di una ristretta cerchia di autori.

Gli storici hanno per molto tempo preferito evitare di mostrare interesse per un tema che - complici le molteplici implicazioni ad esso connesse - avrebbe intaccato la loro immagine di pacati e distaccati osservatori del passato.

Per i cultori del diritto positivo una simile attitudine sarebbe stata difficilmente motivabile: le continuità tra il sistema delle fonti del venten-

\footnotetext{
* Nicola Rondinone, Storia ine-

dita della codificazione civile, $\mathrm{Mi}$ lano: Giuffrè 2003, XVIII, 8I4 p., ISBN 88-I4-IO585-5
} 